\title{
Studying Red Blood Cell Agglutination by Measuring Electrical and Mechanical Properties with a Double Optical Tweezers
}

\author{
A. Fontes*, H. P. Fernandes**, M. L. Barjas Castro**, A. A. de Thomaz*, L. Y. Pozzo*, L. C. \\ Barbosa* and C. L. Cesar* \\ * Instituto de Física Gleb Wataghin - Universidade Estadual de Campinas - 13083-970 - Campinas - \\ SP - Brazil. \\ ** Hematology and Transfusion Center - Universidade Estadual de Campinas - 13083-970 - \\ Campinas - SP - Brazil.
}

The red blood cell (RBC) viscoelastic membrane contains proteins and glycoproteins embedded in, or attached, to a fluid lipid bilayer and are negatively charged, which creates a repulsive electric (zeta) potential between the cells and prevents their aggregation in the blood stream [1]. The first counterions cloud strongly binded moving together with the RBC is called the compact layer [2]. There are techniques, however, to decrease the zeta potential to allow cell agglutination which are the basis of most of the tests of antigen-antibody interactions in blood banks. The identification of the antibodies against the erythrocyte antigens is of fundamental importance for transfusional routines. This report proposes the use of a double optical tweezers [3] for a new procedure for measuring: (1) the apparent membrane viscosity $\left(\eta_{m}\right),(2)$ the cell adhesion $(a),(3)$ the zeta potential $(\zeta)$ and (4) the compact layer's size of the charges formed around the cell in the electrolytic solution $(d)$.

For this experiment, optical tweezers trap a silica beads that binds strongly and non-specifically to RBCs. At the same time, after calibration through its displacement from the equilibrium position using geometrical optics regime, the silica beads acts as a pico-Newton force transducer. To measure the membrane viscosity we trapped silica beads strongly attached to agglutinated RBCs and measured the force to slide one RBC over the other as a function of the relative velocity, as shown in figure 1 (a). The RBC adhesion was measured by slowly displacing two RBCs apart until the disagglutination happens, as shown in figure 1 (b). The compact layer's size was measured using the force on the silica bead attached to a single RBC in response to an applied voltage and the zeta potential was obtained by measuring the terminal velocity after releasing the RBC from the optical trap at the last applied voltage, as shown in figure 2 (a) and 2 (b). The double optical tweezers consisted of a Nd:YAG laser strongly focused through $100 \mathrm{X}$ oil immersion objective. The laser beam is divided once and recombined using polarizer beam-splitters. There is a half-wave plate before the first beam-splitter to distribute the power for each beam. Before the second beam-splitter there is another half-wave plate to control the power of one of the beams. A set of telescopes are used in the system to capture particles in the microscope plane of view. An extra telescope is used for flipping the laser beams without disturbing the alignment. The movements of the beams of each optical tweezers are done by a joystick or computer using piezoelectric actuators of the gimbal mounts. The RBC units were obtained in the Hematology and Transfusion Center of Campinas and diluted $(0.5: 1000 \mu \mathrm{L})$ in $\mathrm{AB}$ human plasma with Rh positive and known refractive index and viscosity. To this solution was added $10 \mu \mathrm{L}$ of silica beads previous diluted in physiological serum.

We obtained a average value of $a=14 \mathrm{pN}$ for the adhesion of RBCs. This measurement was directly quantified using the silica bead displacements from the equilibrium position. For the apparent membrane viscosity we found $\eta_{m}=5.5 \times 10^{-4}$ poise.cm. Using the Smoluchowski equation for the 
terminal velocity, $\mathrm{v}=(\varepsilon E / \eta) \zeta$ (where $\eta$ is the viscosity, $\varepsilon$ is the electrical permittivity of the electrolytic solution and $E$ is the electric field), we found $\zeta=-12.5 \mathrm{~m} \mathrm{~V}$ for the zeta potential. It is also possible to show that the zeta potential can be written as function of the compact layer's size $(d)$ as $F_{o p}=(A \varepsilon \zeta / d) E$, where $A$ is the area of the RBC and $F_{o p}$ is the optical force. Using this equation we found $\mathrm{d}=0.85 \mu \mathrm{m}$ for the compact layer's size. We believe that the results presented in this article demonstrated a new methodology using a double optical tweezers to determine RBC properties such as apparent membrane viscosity, adhesion, zeta potential and the size of the compact layer. The measurements of the zeta potential, as well as the preliminary results of the apparent membrane viscosity, are in agreement with values found in the literature $[4,5,6]$. As far as we know, this is the first time that the size of the compact layer and the RBC adhesion are measured using optical tweezers. The ability of the optical trap to capture and manipulate single particles allowed us to characterize cell by cell individually and also to obtain the zeta potential and the size of the compact layer for the same RBC. Individual cell analysis methods are always more sensitive to small differences than those based on average values. There are various conventional electrophoresis methods to measure cell zeta potential [7]. However, the data acquisition of such methods are not straightforward due to the complex motion of cells inside closed vessels, specially because the velocity of the cell depends on its distance from the walls of the vessels. In conclusion, we believe that the methodology here proposed can provide information about cell agglutination that helps to improve the immunohematologic tests usually performed in blood banks.
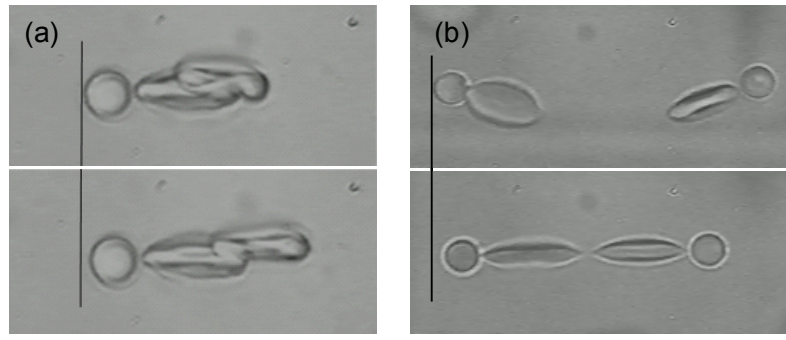

Fig. 1. (a) The measurement of the apparent membrane viscosity. (b) The measurement of cell adhesion using the double optical tweezers.

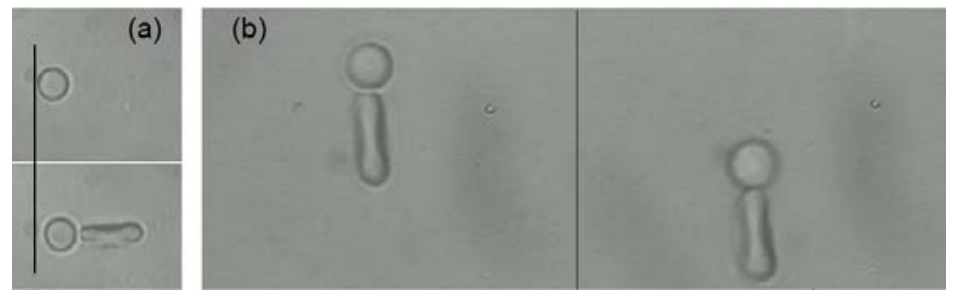

Fig. 2. A example of measurements done in the same cell of the compact layer's size using the optical force (a) and of the zeta potential using the terminal velocity (b).

[1]. W. Pollack and R. P. Reckel, Int. Archs. Allergy Appl. Immun. 54 (1977) 29.

[2]. A. Sze, D. Erickson, L. Ren and D. Li, J. Colloid and Interface Science 261 (2003) 402.

[3]. A. Ashkin and J. M. Dziedzic, Science 235 (1987) 1517.

[4]. R. Hochmuth, P. Worthy and E. Evans, Biophys. J. 26 (1979) 101.

[5]. W. Pollack, H. J. Hager, R. Reckel, D. A. Toren and H. O. Singher, Transfusion 5 (1965) 158.

[6]. C. Zhu, G. Bao and N. Wang, Annu. Rev. Biomed. Eng. 02 (2000) 189.

[7]. N. Hashimoto, S. Fujita, T. Yokoyama, et al., Electrophoresis 19 (1998) 1227. 